\title{
Predictive responses in Parkinson's disease: manual keypresses and saccadic eye movements to regular stimulus events
}

\author{
TREVOR CRAWFORD, SUSAN GOODRICH, LESLIE HENDERSON, \\ CHRISTOPHER KENNARD
}

\author{
From the Department of Neurology, The London Hospital, London
}

SUMMARY In a coincidence timing task, Parkinsonian patients and a control group were instructed to synchronise a keypress with the onset of a visual signal which had been preceded by a regular train of warning signals. Although the Parkinsonian group had previously exhibited slower reactions in a conventional simple reaction-time task, they were able to generate predictive responses that fell as close to the target onset as the controls' but showed greater variability. In a second experiment, Parkinsonian patients and controls made saccadic eye movements to a visual target that stepped at regular intervals between two fixed locations. After a few trials all the subjects tended to make predictive saccades that were initiated before the target excursion. However, the Parkinsonian group were slower to develop this strategy and when they did their saccades became considerably more hypometric than those of the controls. Both groups were able to maintain predictive responding even when the visual target disappeared and responses were paced by a buzzer. We concluded that Parkinsonian patients are capable of initiating predictive responses of the eye and the hand, at least in some circumstances, but such responses tend to be inaccurate in execution. This, in turn, may dispose the Parkinsonian patient against predictive movement.

It has often been suggested that a salient feature of the Parkinsonian deficit of movement initiation is an inability to capitalise on predictabilities in the behaviour of environmental stimuli. A principal source of this generalisation is the classic series of studies of Parkinsonian impairment in tracking tasks, conducted by Flowers. ${ }^{1-6}$ In studies of smooth pursuit and step-tracking, employing somato-motor responses, Flowers reported diverse findings which suggested that Parkinsonian subjects failed to utilise an internal model of the spatio-temporal predictabilities in environmental events to improve performance when tracking regular targets. From these studies, Flowers concluded that "... they have lost the ability to control voluntary movements 'open loop'. ..." However, a difficulty with this view is that the Parkinsonian group, while exhibiting greater phase lag overall, were able to reduce that lag as much as the control group when tracking regular sinusoids. This is

Correspondence to: Professor Henderson, Psychology Division, Hatfield Polytechnic, Hatfield, Hertfordshire AL10 9AB, UK

Received 26 August 1988 and in revised form 29 March 1989. Accepted 6 April 1989 difficult to reconcile with the view that Parkinsonian patients are unable to capitalise on predictabilities. On the other hand, the difference between the groups in tracking error was greater with regular targets. Therefore, an alternative interpretation of these data is that Parkinsonian patients are capable of generating predictive movements but these are peculiarly inaccurate.

In further studies of manual tracking, Flowers ${ }^{5}$ found that patients with Parkinson's disease (PDs) were unable to maintain tracking of repetitive ramps or sawtooths when the target briefly disappeared from the screen. Taken together, these results led Flowers to conclude that either Parkinsonian patients were unable to prepare responses in advance or they were unable to issue such prepared responses unless the movement was summoned by visual events.

Subsequent studies seem to confirm that those with Parkinson's disease have the capacity to act predictively. Bloxham et al $^{7}$ used a continuous, smoothpursuit tracking task and showed that when the target trajectory was changed from a random path to a regular, repetitive one, $P D$ patients could reduce their phase lag as much as normal controls. In a step- 
tracking task where the response is discrete rather than continuously varying, Day et $a l^{8}$ found that PD patients were able to reduce lag when the target was predictable, although not as much as the controls. Moreover, Stelmach et al $^{9}$ found that in a discrete movement, aiming task, partial precuing (hand, direction or extent) speeded the PD patients as much as the controls.

With oculomotor behaviour, there is a concensus regarding the Parkinsonian patients ability to benefit from target predictability when executing smooth pursuit eye movements. Flowers and Downing ${ }^{6}$ contrasted that benefit with the absence of benefit (in accuracy) that they found in manual tracking. Likewise, Bronstein and Kennard ${ }^{10}$ found that the pursuit eye movements of Parkinsonian patients exhibited as much phase lag reduction in response to target predictability as did those of the controls.

The normal function of saccades is to bring the fovea to bear on stimuli that materialise in the visual periphery. In such cases it is often said that the peripheral event (which may be visual, auditory or somato-sensory) "automatically" elicits the appropriate saccadic eye movement. Although this term is unsatisfactory it draws attention to the fact that saccades elicited by new events in the visual periphery appear to have a different status for the movement control system than do saccades which are strategically generated. Saccades of this latter type may be produced in a number of different situations, one of which occurs when an event in the visual periphery is entirely predictable in its location and timing. In this situation, normal subjects may act predictively, generating a saccade to the predicted location without waiting for the arrival of the stimulus. ${ }^{112}$ These saccades frequently precede the target onset. Various studies have shown that PD patients can initiate saccades in the absence of a visual eliciting stimulus, although not as effectively as normal subjects. ${ }^{1314}$
In the following two experiments we questioned whether patients with Parkinson's disease can free themselves from stimulus dependence to the extent of initiating a response in anticipation of an entirely predictable stimulus. In experiment 1 , we attempted to create the most favourable conditions for predictive responding. Subjects were explicitly instructed to try to emit a response precisely coincidental with a target's onset. A countdown procedure was employed to assist with the estimated time of the target's arrival. The response required was simple (depression of a key using the index finger of the preferred hand) and did not require precise calibration. Feedback, immediately after each response, indicated to the subject in a readily assimilated form, the extent and direction of the deviation of that response from zero latency.

\section{EXPERIMENT 1}

\section{Method}

\section{Design}

Originally we had intended that the presence or absence of latency feedback would be a factor in the experiment but when it became obvious that the PD patients could respond predictively almost from the beginning (whether or not they received feedback) we terminated the experiment after data had been collected on five PD patients and six controls.

\section{Subjects}

To meet the criteria for admission to the study the subjects needed to be volunteers whose ages ranged between 50 to 75 years; the controls should be drawn from a panel of elderly subjects with no evidence of CNS disease or impaired movement and who were not taking drugs known to affect CNS function; neither group should show evidence of dementia, as assessed by the Mini Mental States Scale. ${ }^{15}$ In addition, the PD patients were assessed on the Webster ${ }^{16}$ and Hoehn and Yahr ${ }^{17}$ scales of clinical severity (table 1).

\section{Procedure}

Subjects were seated facing a microcomputer-controlled

Table 1 Details of the subjects in Experiment 1

\begin{tabular}{|c|c|c|c|c|c|c|}
\hline \multirow[b]{2}{*}{ Years since initial diagnosis } & \multirow[b]{2}{*}{ Age } & \multirow[b]{2}{*}{ Sex } & \multicolumn{2}{|c|}{ Symptom rating } & \multirow[b]{2}{*}{ Medication } & \multirow[b]{2}{*}{ Feedback? } \\
\hline & & & Webster & Hoehn and Yahr & & \\
\hline \begin{tabular}{ll}
\multicolumn{2}{l}{ Parkinsonian } \\
CO & 4 \\
BR & 6 \\
KE & 1 \\
GI & 2 \\
HA & 3
\end{tabular} & $\begin{array}{l}64 \\
70 \\
64 \\
67 \\
74\end{array}$ & $\begin{array}{l}\mathbf{F} \\
\mathbf{M} \\
\mathbf{M} \\
\mathbf{F} \\
\mathbf{M}\end{array}$ & $\begin{array}{r}6 \\
14 \\
5 \\
3 \\
10\end{array}$ & $\begin{array}{l}\text { Stage II } \\
\text { Stage II } \\
\text { Stage I } \\
\text { Stage I } \\
\text { Stage II }\end{array}$ & $\begin{array}{l}\text { parlodel disipal } \\
\text { tremonil sinemet } \\
\text { sinemet } \\
\text { None } \\
\text { sinemet tremonil }\end{array}$ & $\begin{array}{l}\mathbf{Y} \\
\mathbf{N} \\
\mathbf{N} \\
\mathbf{Y} \\
\mathbf{Y}\end{array}$ \\
\hline $\begin{array}{l}\text { Control Group } \\
\text { SM } \\
\text { KA } \\
\text { HN } \\
\text { HE } \\
\text { KL } \\
\text { WH }\end{array}$ & $\begin{array}{l}58 \\
62 \\
69 \\
64 \\
73 \\
72\end{array}$ & $\begin{array}{l}\mathbf{F} \\
\mathbf{F} \\
\mathbf{M} \\
\mathbf{F} \\
\mathbf{F} \\
\mathbf{F}\end{array}$ & & & & $\begin{array}{l}\mathbf{Y} \\
\mathbf{N} \\
\mathbf{N} \\
\mathbf{Y} \\
\mathbf{Y} \\
\mathbf{Y}\end{array}$ \\
\hline
\end{tabular}


VDU, with the index finger of their preferred hand resting upon a response key. The first task provided a measure of conventional Simple Reaction Time (SRT). Each trial started with a warning signal in the form of a square white cursor appearing in the centre of the screen, for a time randomly varying between 0.75 to 1.5 seconds. This was immediately followed by the imperative signal, the word LEFT or RIGHT according to whichever hand the subject had elected to use. Depressing the response key terminated the display and initiated a two second inter-trial-interval. Subjects were instructed to respond as fast as possible but to avoid anticipatory responses. If the computer recorded an RT of less than 100 msecond a caution was immediately displayed on the screen advising the subject to wait for the signal before responding. After four trials demonstrating the task, 20 data gathering trials were run.

The coincidence timing task then followed. In this task, each trial employed the same countdown sequence, designed to encourage predictive responding, and assist time estimation. On the VDU the stimuli 3-2-1-Go appeared in sequence at a central location, coloured blue, red, yellow and green, respectively. Each countdown stimulus lasted 730 mseconds. The Go signal lasted only $\mathbf{4 0}$ mseconds. Accompanying the onset of each visual stimulus was an audible "beep", lasting 20 mseconds. Subjects were instructed to attempt to depress the response key precisely coincidental with the arrival of the Go signal. The delay between the subject's response and initiation of the next trial sequence was two seconds.

This part of the study comprised five blocks of datayielding trials, following a practice run of four trials to demonstrate the task. Block 1 comprised 20 trials and established an initial baseline level of performance. At the end of block 1, both the group with Parkinson's disease and the control group were randomly divided into subgroups according to whether the subjects were to receive feedback (FB) thereafter or no feedback (NFB). The feedback was designed to be immediately assimilated and to be based on criteria that changed from block to block adaptively, tailoring the reinforcement to the subject's evolving level of performance. Since it turned out that all subjects could learn to anticipate regardless of the feedback, only summary details of the complex feedback algorithm are provided here.

The feedback display comprised a central yellow rectangle with the subscript "BANG ON", flanked on the left and right by red rectangles subtitled "FAST" and "SLOW". Flanking these to left and right were two further red rectangles subtitled "VERY FAST/VERY SLOW". This display followed each response in the feedback condition, with a black arrow travelling from top to bottom of the appropriate rectangle. It remained on the screen throughout the two second inter-trial interval.

The algorithm which determined the category of feedback to be allocated to a response was based on cutpoints derived from the subject's actual distribution of latencies over the preceding blocks. Thus, if the subject improved in a block (with more latencies appoaching zero from either side), for the next block the boundaries of the reinforcement categories would contract toward zero, so as to encourage even more accurate anticipation.

Following block 1, the patients and control FB subgroups received 10 trials, demonstrating the feedback. Data from these trials were not used in the analysis. All four subgroups then received a further four blocks, each consisting of $\mathbf{4 0}$ trials (blocks 2, 3, 4 and 5).

\section{Results and discussion}

The mean latency for each subject is displayed in table 2 , for the conventional SRT task and for each of the five blocks of the coincidence timing task.

From the data on coincidence timing latencies, it seems clear that all the subjects are capable of responding predictively, in the sense that they do not await the arrival of the target (GO) signal before initiating their response. Even in block 1 , before explicit feedback was available, only one (PD) subject showed a mean latency greater than +100 mseconds (positive latency values denote responses that occurred after target onset). Furthermore, this subject (BR) developed negative latencies in the subsequent blocks of trials. There appears to be no relationship between conventional SRT performance and predictive performance.

Table 2 Mean Latencies (msec) in Experiment 1 for the Simple Reaction Time (SRT) and the Coincidence Timing Tasks. (Negative values denote responses made before the onset of the target signal)

\begin{tabular}{|c|c|c|c|c|c|c|c|c|c|c|c|c|}
\hline \multirow[b]{3}{*}{ Ss } & \multirow[b]{3}{*}{$S R T$} & \multicolumn{11}{|c|}{ Coincidence Timing Task } \\
\hline & & \multirow{2}{*}{$\begin{array}{l}\text { NFB } \\
\text { Block } \\
1\end{array}$} & \multicolumn{5}{|c|}{ Feedback (Blocks) } & \multicolumn{5}{|c|}{ No Feedback (Blocks) } \\
\hline & & & 2 & 3 & 4 & 5 & $\bar{x} 2-5$ & 2 & 3 & 4 & 5 & $\overline{\bar{x} 2-5}$ \\
\hline $\begin{array}{l}\text { Parkinson } \\
\text { CO } \\
\text { GI } \\
\text { HA } \\
\text { BR } \\
\text { KE }\end{array}$ & $\begin{array}{l}327 \\
370 \\
557 \\
268 \\
288\end{array}$ & $\begin{array}{r}+60 \\
+\quad 3 \\
+\quad 74 \\
+162 \\
-\quad 7\end{array}$ & $\begin{array}{l}+17 \\
-37 \\
-45\end{array}$ & $\begin{array}{l}+1 \\
-19 \\
-96\end{array}$ & $\begin{array}{l}-3 \\
-54 \\
+15\end{array}$ & $\begin{array}{l}+8 \\
+18 \\
+31\end{array}$ & $\begin{array}{l}+6 \\
-23 \\
-24\end{array}$ & $\begin{array}{r}-237 \\
-\quad 1\end{array}$ & $\begin{array}{r}-129 \\
-\quad 1\end{array}$ & $\begin{array}{l}-86 \\
+20\end{array}$ & $\begin{array}{l}-58 \\
+\quad 7\end{array}$ & $\begin{array}{r}-128 \\
+\quad 6\end{array}$ \\
\hline$\overline{\mathbf{x}}$ & 362 & +58 & -22 & -35 & -14 & +19 & -14 & -119 & -65 & -33 & -26 & -61 \\
\hline $\begin{array}{l}\text { Control } \\
\text { SM } \\
\text { WH } \\
\text { HE } \\
\text { KL } \\
\text { KA } \\
\text { HN }\end{array}$ & $\begin{array}{l}288 \\
327 \\
286 \\
241 \\
341 \\
286\end{array}$ & $\begin{array}{l}-27 \\
+\quad 17 \\
+61 \\
-30 \\
-202 \\
-\quad 27\end{array}$ & $\begin{array}{r}-15 \\
-3 \\
+18 \\
0\end{array}$ & $\begin{array}{r}-19 \\
+9 \\
-6 \\
0\end{array}$ & $\begin{array}{l}-31 \\
-11 \\
-31 \\
+1\end{array}$ & $\begin{array}{r}-13 \\
-19 \\
+22 \\
-19\end{array}$ & $\begin{array}{l}-20 \\
-6 \\
+1 \\
-5\end{array}$ & $\begin{array}{l}-213 \\
-13\end{array}$ & $\begin{array}{l}-79 \\
+\quad 32\end{array}$ & $\begin{array}{l}-66 \\
-26\end{array}$ & $\begin{array}{l}-111 \\
-\quad 23\end{array}$ & $\begin{array}{l}-117 \\
-\quad 8\end{array}$ \\
\hline$\overline{\mathbf{x}}$ & 295 & -35 & 0 & -4 & -18 & -7 & -8 & -113 & -24 & -46 & -67 & -63 \\
\hline
\end{tabular}




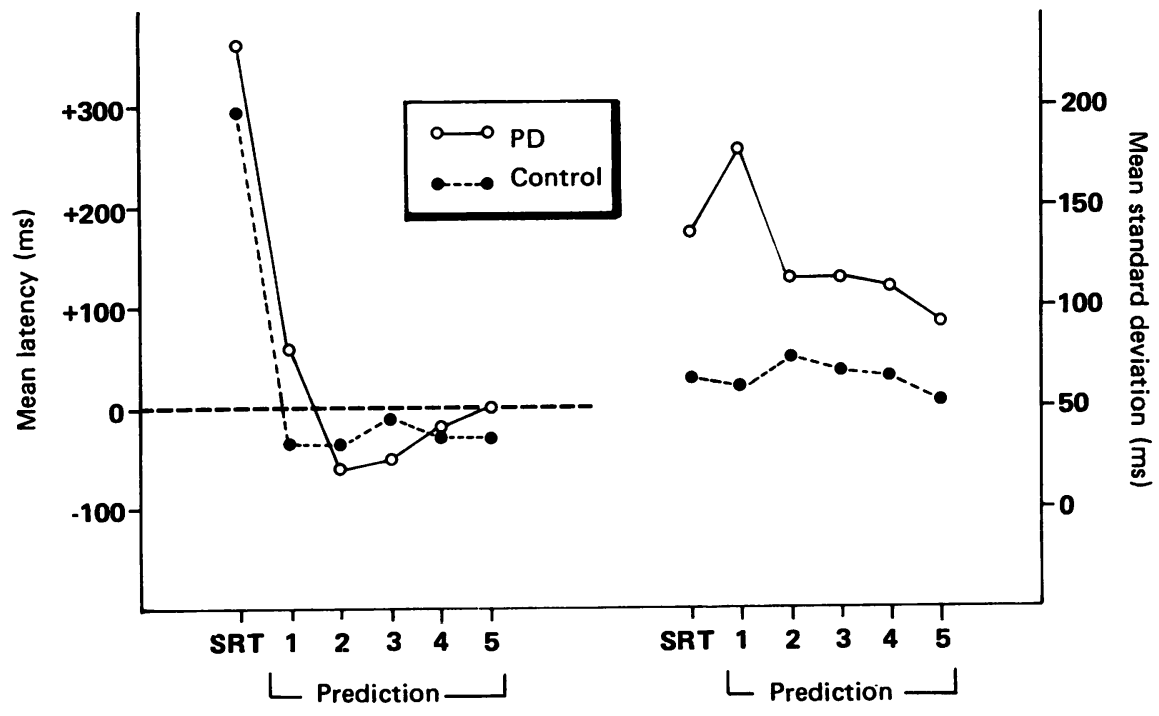

Fig 1 Mean latencies and mean intra-subject standard deviations for the two groups in the Simple Reaction Time task and the 5 blocks of the Coincidence Timing ("Prediction") task.

Blocks of trials

In a coincidence timing task, mean latencies are best regarded as an index of Constant Error, ${ }^{18}$ that is, the tendency of responses to exhibit systematic bias, in terms of falling short of or overshooting the target. In this respect, subjects were remarkably accurate. If we consider the latencies for the individual subjects averaged over blocks $2-5$, we find that nine out of 11 subjects showed values falling within the range +6 to -24 msecond. The remaining two subjects (BR, a patient, and KA, a control) showed very pronounced stimulus-response asynchrony (SRA). While these two subjects did not receive feedback, this was unlikely to be the source of their inaccurate timing, since their SRA values were just as deviant in block 1 , before any of the subjects received feedback.

The paucity of subjects in the No Feedback condition deprived us of any general conclusion regarding the effect of feedback on the accuracy of timing. However, we may safely conclude that in this task explicit feedback is not a necessary prerequisite of accuracy, since subjects $\mathrm{KE}$ and $\mathrm{HN}$ attained high accuracy in the absence of feedback. Indeed, six subjects show SRAs $<30$ mseconds in block 1 , before feedback was introduced. Such mean latencies, considered alone, may give an exaggerated impression of overall accuracy, due to the possibility of large positive and negative SRAs cancelling each other. It is therefore necessary to supplement this measure of Constant Error with a measure of Variable Error. Thus, we calculated the standard deviation of the latencies within a block for each subject.

Figure 1 displays the mean latencies and the mean (intraindividual) standard deviations for the entire Parkinsonian and control groups. Data are shown for the SRT block and for each of the five blocks in the coincidence timing (prediction) task. From these data it can be seen that the individuals within the PD group tend to have more variable latencies than controls. This appears to hold for both tasks. Indeed, the standard deviations are of similar magnitude in the SRT and coincidence timing tasks.
This experiment has established that Parkinsonian patients are capable of responding predictively in a coincidence timing task where a simple, discrete movement is required. This has only been demonstrated for relatively mildly afflicted patients but even those subjects with prolonged SRT latencies could achieve very small stimulus-response asynchronies in the predictive task.

It is not possible to conclude that this severely limits the claim that Parkinsonian patients are stimulus dependent until we can refute the assertion that the patients have merely learnt to transfer their dependence to the last of the sequence of countdown stimuli preceding the GO signal. Since the final? warning signal precedes the GO signal by 730 mseconds the PD patients clearly cannot be approaching it like the imperative signal in a conventional RT task. Their responses follow the onset of the final warning signal by an amount of time that is about twice their average latency in the SRT task. Clearly any anticipation task must involve a signal before the target which serves to set the subject's "clock" running. Accordingly, all we can hope to show is that the subject is able to use a predetermined value derived from that "clock" as an internal cue to initiate a response. What remains to be determined is whether this capacity of Parkinsonian patients for anticipation is restricted to simple uncalibrated responses, such as key pressing, or to situations where a target latency of zero is explicitly specified.

\section{EXPERIMENT 2}

In our second experiment we considered the oculomotor system. Here, a spatially calibrated response was required, so we were able to assess any cost in accuracy that might be associated with anticipation. The task used was developed from that of Teravainen and Calne ${ }^{13}$ who invited subjects to make alternating saccades in the absence of any target, a task which allows neither the assessment of latency nor accuracy. We presented a visual target alternating between fixed points. 
Predictive

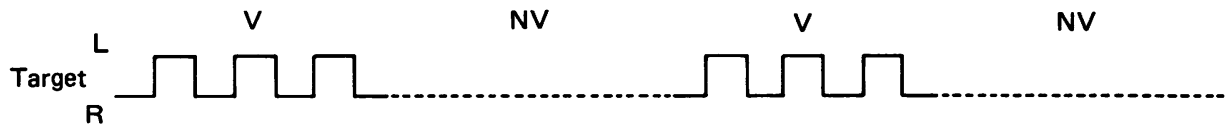

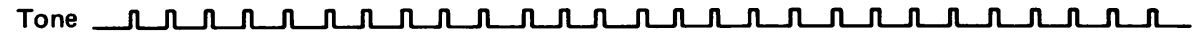

Fig 2 Schematic diagram of the experimental paradigm for eliciting predictive saccades. The target alternated between Left $(L)$ and Right $(R)$ hand, fixed locations, every 2 seconds. The 4 blocks of trials alternated between Vision ( $V$ : tone plus visual target) and No Vision ( $N V$ : tone only) conditions.

By analogy with the tracking through gaps task employed by Flowers ${ }^{5}$ to study manual tracking, the visual targets were periodically withdrawn but an accompanying auditory signal continued to supply temporal information. In contrast to the manual experiment, subjects were merely forewarned that the target would alternate regularly between two fixed locations but their response timing was not directed more specifically.

\section{Method}

Subjects Seven PD patients (four females, three males), and seven controls (four females, three males), who did not have neurological or visual impairment, volunteered to take part in the study. The PD group had a mean age of 61 (range 52-
68 ) and the controls a mean age of 63 (range 53-72). The PD group had mild to moderate motor disability, with one patient rated as Hoehn and Yahr stage III and the remainder as stages I or II. All were taking anti-Parkinsonian drugs at the time of the experiment. None showed evidence of dementia as assessed by the Mini Mental States Scale. None of the controls were taking drugs known to affect CNS function.

Apparatus Eye movements were recorded by an infra-red scleral reflectance device and stimuli were presented by a computer controlled LED display. Full details of the display and the eye movement measurements were provided by Crawford et al. ${ }^{19}$

Procedure Subjects were informed that the target would
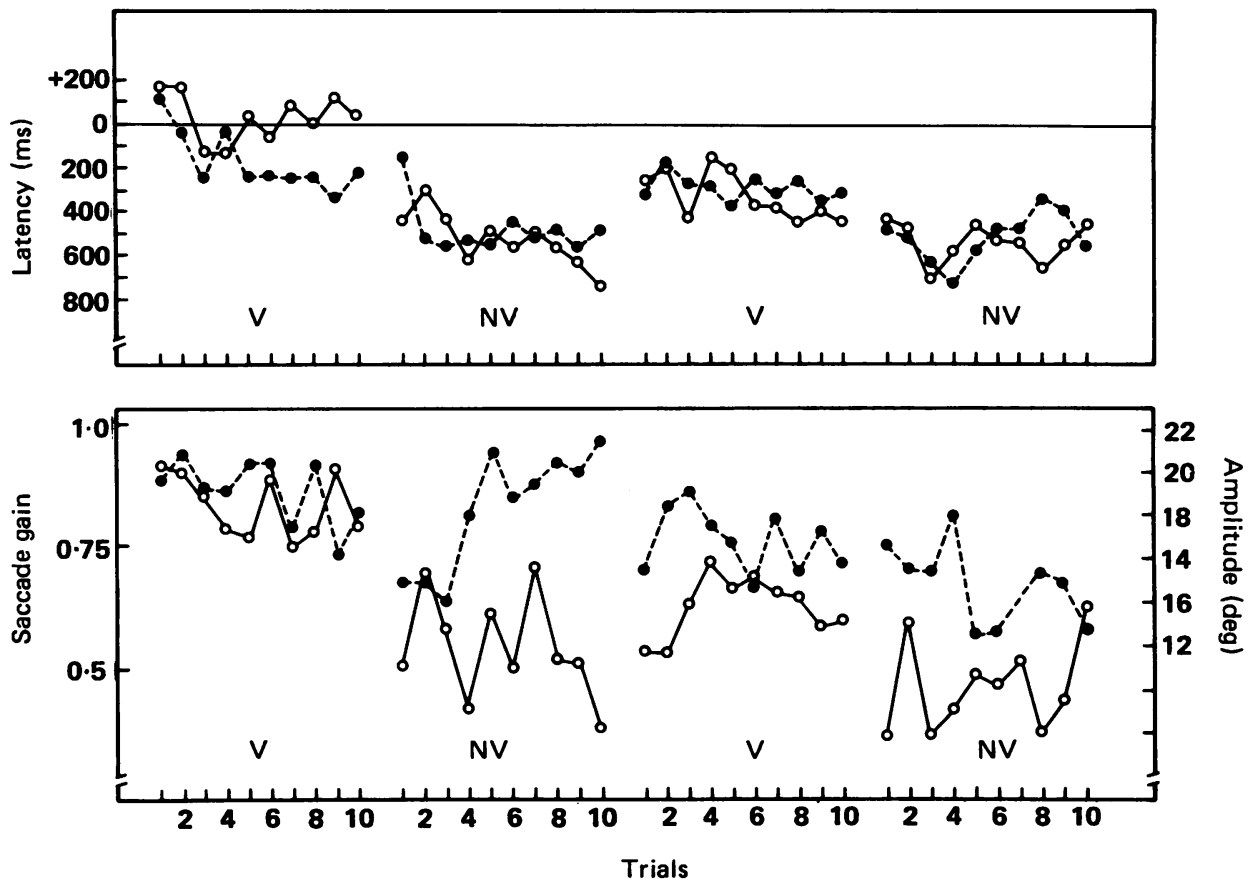

Fig 3 Group mean latency (top panel) and gain/amplitude (bottom panel) of the primary saccade, by trial number and condition. V/NV indicates presence/absence of the visual target. Open circles signify Parkinsonian Groups; filled circles signify controls. 
alternate horizontally between two fixed locations at a fixed rate. They were also advised that there would be four blocks of trials run as a continuous sequence. In blocks 1 and 3 (Condition V) the visual target would alternate back and forth every two seconds and as an extra cue an auditory "beep" would occur coincident with each target onset. In blocks 2 and 4 (Condition NV) the visual target would disappear but the "beeps" would continue as before (see figure 2). They were asked to try to maintain the rate and amplitude of eye movement even in the absence of the targets. The subjects were comfortably seated in a modified dental chair. Head movement was restrained by an adjustable head band attached to the headrest. The room was in total darkness and the target LEDs were the only visible stimuli.

Each of the four blocks of trials comprised 11 target jumps. From a centrally fixed point the first target step was $11 \cdot 25^{\circ}$ to the left. Thereafter the target stepped alternately right and left, through 22.5 degrees every two seconds.

Data analysis Measurements of the latency and amplitude of the primary saccades and of final eye position (FEP) for each trial were obtained by digitising the chart records, using a graphics tablet. The first target step in each tlock was not scored since in block 1 this was a half step and in blocks 2,3 and 4 the first trial signalled the transition to the no vision, vision, no vision conditions respectively. Where blinks and other artifacts prevented the analysis of a trial, the subject's mean value for that block was substituted. Less than $5 \%$ of trial data was lost in this way.

\section{Results}

The latency and amplitude of the primary saccades, trial by trial, is shown in fig 3, for the PD and control groups. From inspection it appears that the pattern of performance obtained in the first block differs from that found in the others, so this block was statistically examined separately. There was no difference between the groups in the amplitude of the primary saccade found in block $1(\mathrm{~F}<1.0)$. In general, responses fell about $10-20 \%$ short of the $22 \cdot 5^{\circ}$ target excursion.

In contrast, the only evidence for any differences in latencies between the groups is to be found in block 1 , where it appears that the control subjects' performance stabilised half way through the block at the anticipatory value which thereafter continues to characterise their latencies with visible targets. However, the PD group, while displaying a mean latency that is far too low to be attributable to them consistently awaiting the target step before responding, stop short of the unequivocally negative latencies that prevail in later blocks. As a simple investigation of this apparent differential trend the latencies in block 1 were partitioned into a first half (trials 1-5) and second half (trials 6-10), and an ANOVA performed with groups and halves as factors. The main effect of the groups did not quite achieve significance $(F=4 \cdot 0$, d.f. $=1,12 ; p<0 \cdot 10$ ), whereas that of the halves was clearly significant $(F=8 \cdot 1$, d.f. $=1,12 ; p<0.01)$. Of greatest interest is the significant interaction $(F=8.9$,
Table 3 Group Means and standard deviations of the final eye positions (in degrees of displacement) for the four blocks. Target displacement was $22.5^{\circ}$

\begin{tabular}{|c|c|c|c|c|c|c|}
\hline & & $\begin{array}{l}\text { BLOCK } \\
\text { CONDITION }\end{array}$ & $\stackrel{l}{V}$ & 2 & $\stackrel{3}{V}$ & $\stackrel{4}{N V}$ \\
\hline $\begin{array}{l}P D \\
\text { Control }\end{array}$ & $\begin{array}{l}\mathbf{X} \\
\text { SD } \\
\mathbf{X} \\
\text { SD }\end{array}$ & & $\begin{array}{r}21.5 \\
1.2 \\
22.4 \\
1.3\end{array}$ & $\begin{array}{r}26.4 \\
4.0 \\
23.6 \\
4.8\end{array}$ & $\begin{array}{r}21.0 \\
1.7 \\
21.4 \\
1.3\end{array}$ & $\begin{array}{r}23.8 \\
6.7 \\
21.3 \\
5.8\end{array}$ \\
\hline
\end{tabular}

d.f. $=1,12 ; p<0.01$ ), indicating that the move towards anticipatory latencies in the second half of block 1 is reliably greater in the controls.

When we examined blocks $2-4$, the groups did not appear to differ in saccadic latency. All the data points in these blocks revealed negative latencies. That is, the primary saccade was initiated substantially in advance of the target step, never less than 100 mseconds, in advance and on average about half a second in advance. Moreover, this tendency to anticipate was consistent with all the subjects. The mean latency collapsed over blocks 2-4 was negative for all 14 subjects.

When blocks 2 and 4 (NV) were collapsed and compared to block $3(\mathrm{~V})$, analysis of variance revealed that the V/NV factor was significant $(F=11 \cdot 8$, d.f. $=1$, $12 ; \mathrm{p}<0.02)$, with earlier response initiation in the $\mathrm{NV} \stackrel{\mathrm{Q}}{\Omega}$ condition. Neither the Groups Factor nor the interac-O tion between the Groups and Conditions approached significance $(F<1)$.

Regarding the accuracy, we find that for the control group, saccadic amplitude appeared to diminish fairly linearly over successive blocks. In order to examine the target conditions with this baseline trend removed, we conducted the analyses of blocks $2-4$ by collapsing together blocks 2 and 4 (NV) and comparing them with block $3(\mathrm{~V})$. The main effect of the target condition on saccadic amplitude did not approach significance. Only the overall difference between groups emerged as significant $(F=5.8$, d.f. $=1,12$; $\mathrm{p}<0.05$ ), indicating that while the PD group showed appreciably more undershoot, this was not reliably qualified by target conditions (V/NV). The undershoot was considerable, with the PD subjects' mean saccadic amplitude in blocks $2-4\left(12 \cdot 7^{\circ}\right)$ being $4^{\circ}$ less than the controls and less than $60 \%$ of the target excursion.

Table 3 supplements the data on primary saccades with information about the average Final Eye Position (FEP) reached on each trial. The groups did not differ reliably in FEP. The salient feature of the data shown is that whereas in the vision condition (blocks 1 and 3) all the subjects' FEPs clustered close to the target amplitude $\left(22.5^{\circ}\right)$ with little variability, in the No Vision condition (blocks 2 and 4) variability was 
considerable, with some subjects consistently coming to rest short of the appropriate location and others continuing past it. Thus the group mean FEP amplitude may convey a spurious impression of accuracy.

\section{Discussion}

These results can be roughly summarised as follows: Patients with Parkinson's disease are capable of voluntary initiation of predictive saccades. However, in comparison with the controls, their initiation of the primary saccade does not become as markedly anticipatory until the visual target has been withdrawn. Thereafter, their latencies become indistinguishable from the controls even when target visibility returns. For both groups, the absence of a visual target provokes earlier initiation of the primary saccade, in anticipation (presumably) of the auditory beep.

The "early departure" that develops in the Parkinsonian group after the visual target is withdrawn is associated with severe hypometria. However, this marked undershoot of the primary saccade does not result from the loss of information about the target amplitude, since it continues even when vision is restored and, moreover, the primary saccade is usually only the first step in a train of miniature saccades which result in a final eye position resembling that of the controls.

Learning to anticipate Under these experimental conditions, the controls seemed to interpret their task as one in which they should successfully transfer their fixation to the location of a predicted target before its arrival. Bronstein and Kennard ${ }^{10}$ have shown that when a randomly stepping target settled down to a predictable alternation, the PD patients and control group's saccadic latencies diminished at a similar rate. However, in a subsequent block of trials where predictability was explicitly guaranteed, the control latencies became distinctly negative (that is, anticipatory), while Parkinsonian latencies remained highly variable but, on average, positive. Even so, almost $30 \%$ of PD latencies were negative. This compared with a value of $60 \%$ for the controls. The percentages of anticipations for block 1 of this study are strikingly similar: $\mathrm{PD}=30 \%$, controls $=63 \%$. In considerable contrast, the percentages for block 2 are $P D=86 \%$, controls $=84 \%$. Thus, whereas the controls learned to anticipate over the first half of block 1, it was not until the visual target was withdrawn at the beginning of block 2 that PD subjects consistently adopted an "early departure" strategy. We insert the qualification "consistently" because only one PD subject showed no negative latencies whatsoever in block 1 . Whereas only one PD subject showed a majority of negative latencies in block 1 , in block 2 only one PD subject failed to do so. As figure 3 shows, the withdrawal of visual information immediately impels the PD subjects into early departure, with latencies indistinguishable from the controls after the beginning of block 2 .

It appears that two aspects of the latency data require explanation: (i) why do the $\mathrm{PD}$ patients not develop an early departure strategy until the visual target is withdrawn? (ii) why thereafter is the departure time for both groups later, although still anticipatory, when the visual target is present than when it is absent?

As a preliminary to our explanation, we require to distinguish between various roles that may be played by a visual target. First, when a novel stimulus appears away from the point of fixation its onset automatically attracts a saccade, quasi-reflexively. In electing to generate predictive eye movements that anticipate the stepping of the target to its peripheral location, the subject foregoes this automatic, saccade-eliciting role of the target. As we shall see later this may have crucial consequences for saccadic accuracy in the PD group. Secondly, when a saccade is initiated in anticipation of the target step, the visual stimulus plays another role, which is that of a continuing stimulus at the point of fixation, away from which the eye movement has to be made. Concerning this role we postulate a difficulty in releasing fixation from the "attentional capture" exercised by an enduring foveal stimulus. Posner ${ }^{20}$ has shown that attention is usually shifted to a new location before the execution of the saccade to that location. Furthermore, Mayfrank et al ${ }^{21}$ and Ross and Ross $^{22}$ have shown that pre-releasing attention from the central fixation point by extinguishing it shortly prior to arrival of the novel peripheral target (the "gap paradigm"), leads to an increase in the proportion of very short latency "express" saccades.

We suggest that this "attentional capture" effect is responsible for the slight retardation of saccade initiation shown by both groups in block 3 (Vision) as compared to blocks 2 and 4 (No Vision). It appears that persistence of the fixation stimulus only exercises this effect when there is no new peripheral target to summon the saccade, since we have found that when the fixation point persists after the arrival of a peripheral target (the "overlap" paradigm), saccadic latencies were only negligibly prolonged, and any effect of fixation point persistence was equal for PD patients and controls. ${ }^{19}$

A possible reason why it takes the PD patients longer to settle down to consistent early departure is because the group are merely slow to learn an anticipatory strategy. In support of this it might be argued that the subjects need to learn to free themselves from a "passive" reaction to the visual target's displacement. Since patients with Parkinson's disease have been shown to display less strategic flexibility in a number of attention switching and problem solving 
tasks, ${ }^{23-26}$ it is plausible that their development of an alternative anticipatory strategy might be retarded. While this hypothesis remains in contention, we favour an account which attempts to embrace several different aspects of the data. In particular, our preferred account links the latency trends with the accuracy data and offers an explanation of why both of these show a sharp transition for the PD group when the visual target is removed at the beginning of block 2 .

So far, we have considered two roles that the visual stimulus may play at the time of initiating the saccade; that of a peripheral eliciting target and, in predictive responding, that of a persistent fixation target from which attention must be released before the new saccade can be made. However, in the visual target condition, the stimulus may play a third role, providing external feedback on the distance off target after execution of the primary saccade. It is the availability of this feedback in block 1 which we believe is responsible for inhibiting the PD group's adoption of a consistent anticipatory strategy. This is because the PD patients' saccades become severely hypometric when anticipatory (fig 3 ). This is true of the occasional anticipatory saccades made in block 1 . For example, although the mean saccade amplitude for PD patients in block 1 was $18.6^{\circ}$, the mean for each subject's earliest departure saccade (all but one, negative in latency) was only $16 \cdot 3^{\circ}$. Therefore, the withdrawal of vision at the inception of block 2 allows the PD patients to learn to neglect the cost in accuracy which they incur when responding predictively. Of course the data on Final Eye Position show that internal cues still provide information about target location but we assume that internally generated error information has less impact on the saccade initiating strategy.

Anticipation? We have argued that patients with Parkinson's disease, in this task, are capable of responding in anticipation of a stimulus without their response being summoned directly by an external cue. Furthermore, this predictive behaviour is the more remarkable in the domain of saccadic eye movements because it requires a target-driven quasi-reflexive mode of responding to be abandoned. We suggest, therefore, that attempts to explain the failure of patients with Parkinson's disease to act predictively in terms of a deficit in the motor control system - such as inability to drive responses with a mental model of environmental regularities ${ }^{27}$-are inappropriate. It would be more productive to pursue the question of what commonly disposes the Parkinsonian against a predictive strategy.

The Parkinsonian Hypometria In this study our primary interest lay in response timing but several aspects of the metrics of the eye movements merit discussion.
With the data from the controls, we found a progressive increase in saccadic error over successive blocks. This consisted of an undershoot by the primary saccade that increased irrespective of target conditions. This trend is atypical of normal saccadic performance, even in tasks that require a deliberately initiated saccade (for example, Crawford et al ${ }^{19}$ using the same subjects). We ascribe it to an evolving strategy whereby the subjects construe their task as one of arriving at the next target location in advance of that target, whilst becoming increasingly indifferent to the proportion of this traverse that is achieved by the primary saccade. Hence, the primary saccade reflects increasing hypometria whereas the final eye position achieved does not change appreciably.

Two aspects of the amplitude data for the PD patients are striking. In block 1 , no difference between the groups was seen and the undershoot was well within the normally expected range of saccadic gain. In contrast, from the beginning of block 2 onwards the PD group showed severe hypometria, significantly in excess of that found for the controls. Our account of these effects links them causally with the associated effects we have described in the latency data.

We have already suggested that the PD group do not readily develop a consistently predictive strategy during block 1. Moreover, in that block once the targeto arrived, their occasional anticipatory responses were likely to be met with the external evidence that theiro primary saccade was considerably hypometric.

The removal of the visual target at the beginning of block 2 immediately freed the PD patients from the impact of external, visual feedback about the con sequences of their ventures into predictive responding. At the same time, the withdrawal of vision removes the obstacle to anticipatory responding caused by the "inertial" effect encountered when attempting a saccade away from a persisting fixation stimulus without being able to transfer attention to the arrival of a novel peripheral target (what we called "attentional capture" by a fixation stimulus). We were surprised at the abruptness of the transition to a consistently anticipatory strategy in the PD group once vision is removed and the maintenance of that new strategy after the visual target returns in block 3. The PD group may have persisted in predictive responding even after the visual target returned in block 3 because their train of saccades was by that stage initiated sufficiently early that by the time the target arrived the discrepancy between the eye and target position had been reduced to tolerable proportions.

To complete this account we need to explain why patients with Parkinson's disease incur a penalty in terms of saccadic undershoot when they adopt a predictive strategy. We attributed this to the existence of two saccadic control systems. One of these is 
exclusively dedicated to saccades that are elicited by novel spatial targets. This system is spared, at least in mild Parkinsonism. The other system deals with saccades that are not elicited by novel targets, whether they be deliberately produced saccades to a remembered target location ${ }^{1928}$ or predictive saccades that anticipate target arrival, as in this study. The control system responsible for this second class of saccades appears to be distinguished neuro-anatomically by involving the frontal eye fields and substantia nigra pars reticulata. However, previous evidence for both the frontal ${ }^{29}$ and the basal ganglia ${ }^{19} 3031$ involvement has been confined to the "remembered" saccade and the "antisaccade" paradigms. Our finding that the execution of predictive saccades is also selectively impaired in a basal ganglia disorder extends our understanding of the functional role of this secondary control system.

\section{Conclusion}

There is no doubt that subjects with mild Parkinson's disease are able to generate predictive responses in the tasks in our experiments. In the simple key press study, which was explicitly presented as a coincidence timing task, but lacked a spatial accuracy component, the PD group shifted from a "reactive" to a "predictive" mode of responding as readily as the controls. Even PD subjects with relatively retarded simple RT were able to generate near zero mean latencies. Intrasubject variability of latency was, however, higher in the PD group, in both reactive and predictive tasks.

In the saccadic task, where subjects were allowed to evolve their own strategies without explicit direction, the PD group were slower to develop consistently anticipatory responses. When such responses did develop they were accompanied by hypometria. We have suggested an account of the Parkinsonian resistance to the adoption of a predictive strategy as an automatic adaptation to their loss of accuracy for anticipatory saccades.

What remains to be seen is whether some unified account can be given of Parkinsonian impairments that hold across the oculomotor and somatomotor domain. For the saccadic system, the responses that are spared regarding accuracy are those directly elicited by novel peripheral targets. This exact condition is unlikely to have generality because the saccadic system is functionally adapted to cope with precisely those sorts of stimuli. This is reflected in the automatic nature of such eye movements. However, perhaps this spared oculomotor system finds its somatomotor equivalent in clinical reports of kinesia paradoxica where, for example, an otherwise akinetic patient is able to catch a suddenly thrown object.

It is not helpful to characterise the Parkinsonian impairment in general merely as an abnormal dependence on visual information. Visual information may play several distinct roles in the control of action, specifying spatial targets, providing the imperative signal summoning a response, furnishing feedback of the relation between effector and target, and the final knowledge of results. We know of no systematic attempt to adjudicate between these factors, as they affect the Parkinsonian deficit. (In Experiment 2, it was the withdrawal of visual information that was associated with the development of anticipatory responding.)

Finally, one strand of evidence that suggests it is worth attempting an account which subsumes the oculomotor and somatomotor domains is that the deficit in response execution takes the same form in each, namely a train of miniature responses where a single, fast excursion would normally be expected.

This research was supported by grants from the Medical Research Council, the Parkinson's Disease Society and the Wellcome Trust. We thank Christian Lueck for helpful comments on a draft, Rabin Ezra for the computer programming, Malcolm Hawken for technical advice and Tom Koeze for the use of his digitising tablet.

A short account of Experiment 2 is included in Crawford et al. ${ }^{28}$

\section{References}

1 Flowers KA. Ballistic and corrective movements on an aiming task: intention tremor and parkinsonian movement disorders compared. Neurology 1975;25:413-21.

2 Flowers KA. Visual "closed-loop" and "open-loop" characteristics of voluntary movement in patients with parkinsonism and intention tremor. Brain 1976;99:261-310.

3 Flowers KA. The predictive control of behaviour: appropriate and inappropriate actions beyond the input in a tracking task. Ergonomics 1978;25:176-91.

4 Flowers KA. Some frequency response characteristics of Parkinsonism on pursuit tracking. Brain 1978;101:19-34.

5 Flowers KA. Lack of prediction in the motor behaviour of Parkinsonism. Brain 1978;101:35-52.

6 Flowers K, Downing AC. Predictive control of eye movements in Parkinson's disease. Ann Neurol 1978;4:63-6.

7 Bloxham CA, Mindel TA, Frith CD. Iritiation and execution of predictable and unpredictable movements in Parkinson's disease. Brain 1984;107:371-84.

8 Day BL, Dick JPR, Marsden CD. Patients with Parkinson's disease can employ a predictive motor strategy. $J$ Neurol Neurosurg Psychiatry 1984;47:1299-306.

9 Stelmach GE, Worringham CJ, Strand EA. Movement preparation in Parkinson's disease: the use of advance information. Brain 1986;109:1179-94.

10 Bronstein AM, Kennard C. Predictive ocular motor control in Parkinson's disease. Brain 1985;108:925-40.

11 Polidora VJ, Ratoosh P, Westheimer G. Precision of rhythmic responses of the oculomotor system. Percept Mot Skills 1957; 7:247-50. 
12 Stark L, Vossius G, Young LR. Predictive control of eye tracking movements. IRE Trans on Human Factors in Electronics, H62, HFE2, 52-57.

13 Teravainen H, Calne DB. Studies of Parkinsonian movement. 1. Programming and execution of eye movements. Acta Neurol Scand 1980;62:137-48.

14 White OB, Saint-Cyr JA, Tomlinson RD, Sharpe JA. Ocular motor deficits in Parkinson's disease. II. Control of the saccadic and smooth pursuit systems. Brain 1983;106:571-87.

15 Folstein MF, Folstein SE, McHugh PR. "Mini-Mental State"-A practical method for grading the cognitive state of patients for the clinician. J Psychiatr Res 1975;12:189-98.

16 Webster DD. Critical analysis of disability in Parkinson's disease. Modification Treatment 1968;5:257-82.

17 Hoehn MM, Yahr MD. Parkinsonism: onset, progression and mortality. Neurology 1967;17:427-42.

18 Schmidt RA. Motor Control and Learning: A Behavioural Emphasis. Champaign: Human Kinetics Publishers, 1982.

19 Crawford T, Henderson L, Kennard C. Abnormalities of nonvisually guided eye movements in Parkinson's disease. Brain 1989 , in press.

20 Posner MI. Orienting of attention. J Exper Psychol 1980;32:3-25.

21 Mayfrank L, Mobashery M, Kimmig H, Fischer B. The role of fixation and visual attention in the occurrence of express saccades in man. Eur Arch Psychiatry Neurol Sci 1986;235: 269-75.

22 Ross LE, Ross SM. Saccade latency and warning signals: stimulus onset, offset and change as warning signals. Percept Psychophysics 1980;27:251-7.

23 Brown RG, Marsden CD. Internal versus external cues and the control of attention in Parkinson's disease. Brain 1988;111: $323-45$.

24 Cools AR, Van den Bercken JHL, Horstink MWI, Van Spandonck KPM, Berger HJC. Cognitive and motor shifting aptitude disorder in Parkinson's disease. J Neurol Neurosurg Psychiatry 1984;47:443-53.

25 Flowers KA, Robertson C. The effect of Parkinson's disease on the ability to maintain a mental set. $J$ Neurol Neurosurg Psychiatry 1985;48:517-29.

26 Lees AJ, Smith E. Cognitive deficits in the early stages of Parkinson's disease. Brain 1983;106:257-70.

27 Stern Y, Mayeux R, Rosen J, Ison J. Perceptual motor dysfunction in Parkinson's disease: a deficit in sequential and predictive voluntary movement. J Neurol Neurosurg Psychiatry 1983;46: 145-51.

28 Crawford TJ, Henderson L, Kennard C. The saccadic dysmetria in Parkinson's disease is stimulus dependent. In: Crossman AR, Sambrook MA, eds. Neural Mechanisms of Disorders of Movement. London: John Libbey, 1989.

29 Guitton D, Buchtel HA, Douglas RM. Frontal lobe lesions in man cause difficulties in suppressing reflexive glances and in generating goal directed saccades. Exp Brain Res (Berlin) 1985;58: 455-72.

30 Hikosaka $\mathrm{O}$, Sakamoto $M$. Cell activity in monkey caudate nucleus preceding saccadic eye movements. Exp Brain Res (Berlin) 1986;63:659-62.

31 Hikosaka O, Wurtz RH. Modification of saccadic eye movements by GABA-related substances. II. Effects of muscimol in monkey substantia nigra pars reticulata. J Neurophysiol 1985;53 292-308. 